\title{
Determining all (2,3)-torus structures of a symmetric plane curve
}

\author{
Remke Kloosterman
}

\begin{abstract}
In this paper, we describe all $(2,3)$-torus structures of a highly symmetric 39-cuspidal degree 12 curve.

A direct computer-aided determination of these torus structures seems to be out of reach. We use various quotients by automorphisms to find torus structures. We use a height pairing argument to show that there are no further structures.
\end{abstract}

\section{Introduction}

Let $f \in \mathbf{C}[u, v, w]$ be a reduced non-constant homogeneous polynomial and denote with $C \subset \mathbf{P}^{2}$ the corresponding plane curve. A $(p, q)$-torus structure (or $(p, q)$-torus decomposition) of the curve $C$ consists of two homogeneous polynomials $g, h \in \mathbf{C}[u, v, w]$ such that $g^{p}+h^{q}=f$, a $(p, q, r)$-quasi-torus structure consists of three homogeneous polynomials $(g, h, k)$ such that $g^{p}+h^{q}=k^{r} f$ and $\operatorname{gcd}(g, h, k)=1$.

The $(2,3,6)$-quasi-torus structures correspond to the non-identity elements in the group $E(\mathbf{C}(s, t))$, i.e., the $\mathbf{C}(s, t)$-points on the curve $E$ given by

$$
y^{2}=x^{3}+f(s, t, 1) .
$$

The group $E(\mathbf{C}(s, t))$ is finitely generated and torsion-free (due to our assumptions on $f$ ). Cogolludo-Agustín and Libgober [1] showed that for a certain class of plane curves (including curves with at most $A_{1}$ and $A_{2}$ singularities) the rank of this group equals twice the vanishing order at a sixth primitive root of unity of the Alexander polynomial of the curve $C$. A precise description of the class of reduced

The author has been supported by the GNSAGA of INDAM. The author gratefully thanks Carel Faber, Matthias Schütt and the referee for the constructive comments and recommendations which definitely helped to improve the readability of the paper. 
plane curves for which this result holds and similar results for other values of $(p, q, r)$ can be found in [4].

Given a plane curve one can ask the following two questions:

1. Determine the number of $(2,3)$-torus structures.

2 . Do the $(2,3)$-torus structures generate the group $E(\mathbf{C}(s, t))$ of $(2,3,6)$-quasitorus structures?

For cuspidal curves of degree 6 we answered both questions in [4]. The first question was answered using a height pairing on $E(\mathbf{C}(s, t))$. It turned out that the answer to the second question is "yes" for each cuspidal plane curve. D.T. Pho considered the case of the 9-cuspidal curve in [6]. For other geometric interpretations of $(2,3)$-torus structures and their relevance for singularity theory, we refer to [2].

In general, the answer to the second question may be negative: in [4] we constructed a degree 12 plane curve such that the rank of $E(\mathbf{C}(s, t))$ is two, but this curve does not admit any $(2,3)$-torus structures.

For most cuspidal curves of degree 12 , one can easily determine all $(2,3)$-torus structures, sometimes using the results for degree 6 curves. A notable exception is the degree 12 curve with 39 cusps. To the author's knowledge, there is only one family of such curves described in the literature. This is a four-dimensional family. It contains a two-dimensional subfamily of curves, which are $\mathbf{Z} / 3 \mathbf{Z} \times \mathbf{Z} / 3 \mathbf{Z}$-covers of the three-cuspidal quartic curve. These curves play an important role in Hirano's construction of curves with many cusps [3].

In this paper, we consider only one member of this family:

$$
\begin{aligned}
f= & -v^{12}-w^{12}-3 v^{6} w^{6}-2 v^{3} w^{9}-2 v^{9} w^{3} \\
& +\left(-12 v^{3} w^{6}-12 v^{6} w^{3}+12 w^{9}+12 v^{9}\right) u^{3} \\
& +\left(-42 v^{6}-42 w^{6}+138 v^{3} w^{3}\right) u^{6}+\left(36 w^{3}+36 v^{3}\right) u^{9}+27 u^{12}
\end{aligned}
$$

This curve has an additional automorphism, switching $v$ and $w$. In [1] it is shown that the Alexander polynomial of this curve equals $\left(t^{2}-t+1\right)^{4}$, hence the MordellWeil rank is eight. Using a deformation argument, one can show that each curve in the above mentioned family with 39 cusps and no further singularities has Alexander polynomial $\left(t^{2}-t+1\right)^{4}$.

Consider a $(2,3)$-torus structure $h^{2}+g^{3}=f$ of $C$. If we multiply $g$ with a third root of unity and $h$ with 1 or -1 then we obtain again a $(2,3)$-torus structure. Hence there is a natural $\mu_{6}$-action on the torus structures. Two torus structures are equivalent if they are in the same $\mu_{6}$-orbit. We show the following result.

Theorem 1.1. The curve $\{f=0\}$ admits precisely 6 inequivalent $(2,3)$-torus structures and the torus structures generate the Mordell-Weil group.

Moreover, we will give explicit expressions for these torus structures. 
In order to determine all $(2,3)$-torus structures, we could take generic polynomials $g, h \in \mathbf{C}[u, v, w]$ of degree 6 and 4 and consider the coefficients $u^{i} v^{j} w^{12-i-j}$ of

$$
g^{2}=h^{3}+f
$$

This gives 91 equations in 43 unknowns. We could try to ask a computer algebra package like Singular to solve this system. The main step is then to find a Groebner basis for the ideal defined by these 91 equations. We aborted an attempt to do this, because of the long run time.

In this paper, we exploit the existence of the automorphisms to determine all torus structures: Let $G$ be a subgroup of the automorphism group of $C$ and let $\tilde{f}=0$ be a plane model for $C / G$. We now study the Mordell-Weil group of $y^{2}=x^{3}+\tilde{f}(s, t, 1)$. Since $\tilde{f}$ has lower degree or is significantly more singular we can easily find torus structures for $\tilde{f}$. We can then pull these back to $C$.

By varying $G$ we find four inequivalent torus structures (hence 24 in total). A simple argument using the action of the automorphism group on these structures shows that these torus structures generate a rank 8 subgroup $\Lambda$ of the Mordell-Weil group, hence $\Lambda$ is of finite index.

We then consider the height pairing on $\Lambda$. In [4] we showed that the height pairing is integral. We determine all vectors $v \in \Lambda \otimes \mathbf{Q}$ such that the height pairing on $\Lambda+\mathbf{Z} v$ is integral. For each such $v$ we then use explicit formulas for the group law on $E$ to show that $v$ does not correspond to a point in $E(\mathbf{C}(s, t))$ unless $v \in \Lambda$. This shows that $\Lambda$ is the Mordell-Weil group, and that $E(\mathbf{C}(s, t))$ is generated by torus structures.

The torus structures correspond to the vectors with length 4 in $\Lambda$. It is a straight-forward exercise to find all length 4 vectors in a given positive definite integral lattice. It turns out that we have precisely 36 such vectors.

Finally, note that $f \in \mathbf{Q}[u, v, w]$. Hence, we also have a $\operatorname{Gal}(\overline{\mathbf{Q}} / \mathbf{Q})$-action on the torus structures. In the second part, we describe the Galois action. We show that for $y^{2}=x^{3}-1296 f$ all points in $E(\mathbf{C}(s, t))$ have coordinates from $E(\mathbf{Q}(\omega)(s, t))$, where $\omega$ is a primitive third root of unity. Moreover, we show that $E(\mathbf{Q}(s, t))$ has rank 4 and we give generators for $E(\mathbf{Q}(s, t))$.

\section{Mordell-Weil group}

Let $E / \mathbf{C}(s, t)$ denote the elliptic curve

$$
y^{2}=x^{3}-1296 f(s, t, 1) .
$$

The factor -1296 simplifies several formulas later on. 
Since $f$ is irreducible and its degree is divisible by 6 , we have $E(\mathbf{C}(s, t))$ is torsion-free. Since the Alexander polynomial of $f=0$ is $\left(t^{2}-t+1\right)^{4}[1$, Example 6.3] we have $\operatorname{rank} E(\mathbf{C}(s, t))=8$. Moreover, as an elliptic curve it has complex multiplication by $(x, y) \mapsto(\omega x, y)$, where $\omega^{2}=-\omega-1$. In particular, $E(\mathbf{C}(s, t))$ is a free $\mathbf{Z}[\omega]$-module of rank 4 .

Take now the following $x_{i}$

$$
\begin{aligned}
& x_{1}=36 u\left(u^{3}+w^{3}\right) \\
& x_{2}=36 u\left(u^{3}+v^{3}\right) \\
& x_{3}=-12\left(\omega^{2} v^{4}-\omega v^{3} w-\omega^{2} v w^{3}+\omega w^{4}-3 \omega^{2} u^{3} v-3 \omega u^{3} w+3 v^{2} w^{2}\right) \\
& x_{4}=-12\left(\omega^{2} w^{4}-\omega w^{3} v-\omega^{2} w v^{3}+\omega v^{4}-3 \omega^{2} u^{3} w-3 \omega u^{3} v+3 v^{2} w^{2}\right) \\
& x_{5}=36 u\left(7 u^{3}-v^{3}-w^{3}\right) \\
& x_{6}=12\left(3 u^{3} v+3 u^{3} w-v^{4}+v^{3} w-3 v^{2} w^{2}+v w^{3}-w^{4}\right)
\end{aligned}
$$

Moreover, let

$$
y_{1}=36\left(3 u^{6}-6 u^{3} v^{3}+v^{6}+12 u^{3} w^{3}+v^{3} w^{3}+w^{6}\right)
$$

and let $y_{2}$ be obtained from $y_{1}$ by interchanging $v$ and $w$.

We have that $x_{4}^{3}-1296 f(u, v, w)$ is a square, say $y_{3}^{2}$, with $y_{3} \in \mathbf{C}[u, v, w]$. Let $y_{4}$ be obtained from $y_{3}$ by interchanging $v$ and $w$ in $y_{3}$. A direct calculation shows that $y_{3}, y_{4} \in \mathbf{Q}(\omega)[u, v, w]$.

Denote $P_{i}:=\left(x_{i}(s, t, 1), y_{i}(s, t, 1)\right)$.

Using the formula for the group law on $E$ one easily checks that $x_{5}$ is the $x$-coordinate of $P_{1}+P_{2}$ and $x_{6}$ is the $x$-coordinate of $P_{3}+P_{4}$. Denote with $y_{5}$ and $y_{6}$ the respective $y$-coordinates.

The formulas for $y_{3}, y_{4}$ and $y_{6}$ are slightly more complicated than the formulas for the $x$-coordinates. Therefore, we choose not to include them.

Proposition 2.1. We have that

$$
E(\mathbf{C}(s, t))=\oplus_{i=1}^{4} \mathbf{Z}[\omega] P_{i}
$$

Proof. We start by determining the height pairing (as introduced in [4]) on the set $\left\{P_{i}, \omega P_{i} \mid i=1, \ldots, 4\right\}$.

From [4, Section 5] it follows that the heights of the $P_{i}$ and $\omega P_{i}$ are 4 , and that the pairing of $P_{i}$ with $\omega P_{i}$ is -2 . Moreover, the same results give a formula for the pairing of $\omega^{i} P_{k}$ and $\omega^{j} P_{m}$ in terms of the degree of the gcd of $\omega^{i} x_{k}-\omega^{j} x_{m}$ 
and $y_{k}-y_{m}$. This is straightforward and we obtain the following Gram matrix

$$
A:=\left(\begin{array}{cccccccc}
4 & -2 & -2 & 1 & 0 & 0 & 0 & 0 \\
-2 & 4 & 1 & -2 & 0 & 0 & 0 & 0 \\
-2 & 1 & 4 & -2 & 0 & 0 & 0 & 0 \\
1 & -2 & -2 & 4 & 0 & 0 & 0 & 0 \\
0 & 0 & 0 & 0 & 4 & -2 & -2 & 1 \\
0 & 0 & 0 & 0 & -2 & 4 & 1 & -2 \\
0 & 0 & 0 & 0 & -2 & 1 & 4 & -2 \\
0 & 0 & 0 & 0 & 1 & -2 & -2 & 4
\end{array}\right)
$$

The determinant of this matrix is $3^{8}$. In particular, the $P_{i}$ and $\omega P_{i}$ generate a rank 8 group. Hence, these points generate a sub-lattice of finite index in the Mordell-Weil lattice.

Suppose the $P_{i}$ generate a proper sub-lattice of the Mordell-Weil lattice then there would be a vector $v=\left(a_{1}, \ldots, a_{8}\right) \in \mathbf{Q}^{8}$ such that the height of

$$
a_{1} P_{1}+a_{2} \omega P_{1}+\ldots+a_{8} \omega P_{4}
$$

is integral, and the height pairing with each of the $P_{i}$ and each of the $\omega P_{i}$ is integral.

In particular, A.v has integral entries. Using that the entries of $A^{-1}$ are in $1 / 9 \mathbf{Z}$ (or using that the lattice is a direct sum of two lattices of discriminant $3^{4}$ ) we obtain that for each $i$ we have $9 a_{i} \in \mathbf{Z}$.

One easily checks that if $\left(a_{1}, a_{2}, a_{3}, a_{4}\right)$ are not all integral, but $A . v$ is integral then there exists $r, s, t, \in \mathbf{Z}$ such that

$$
\left(a_{1}, a_{2}, a_{3}, a_{4}\right) \equiv \frac{1}{9}(4 r+6(s+t), 2 r+3 s, 2 r+3 t, r) \bmod \mathbf{Z} .
$$

Similarly

$$
\left(a_{5}, a_{6}, a_{7}, a_{8}\right) \equiv \frac{1}{9}\left(4 r^{\prime}+6\left(s^{\prime}+t^{\prime}\right), 2 r^{\prime}+3 s^{\prime}, 2 r^{\prime}+3 t^{\prime}, r^{\prime}\right) \bmod \mathbf{Z}
$$

Also $v^{T} A v$ has to be integral. A straightforward calculation shows that this is

$$
\frac{2}{9}\left(6 s^{2}+9 s t+6 s r+6 t^{2}+6 t r+2 r^{2}+6 s^{2}+9 s^{\prime} t^{\prime}+6 s^{\prime} r^{\prime}+6 t^{\prime 2}+6 t^{\prime} r^{\prime}+2 r^{2}\right)
$$

In particular $r^{2}+r^{\prime 2}$ is divisible by 3 . Since 2 is not a square modulo 3 , the only possibility is that both $r$ and $r^{\prime}$ are divisible by 3 . Replacing $r$ by $3 r$ and $r^{\prime}$ by $3 r^{\prime}$ and considering the above quantity modulo integers reduces the above formula for height to

$$
\frac{1}{3}\left(s^{2}+t^{2}+\left(s^{\prime}\right)^{2}+\left(t^{\prime}\right)^{2}\right)
$$


modulo integers. Hence either all four of $\left\{s, t, s^{\prime}, t^{\prime}\right\}$ are zero modulo three, or exactly one of them is zero modulo 3 .

We did not use the $\omega$-action and -1 action until now. Since $\omega$ sends the vector $\left(r, s, t, r^{\prime}, s^{\prime}, t^{\prime}\right)$ to $\left(r+t, s, t, r^{\prime}+t^{\prime}, s^{\prime}, t^{\prime}\right)$ and -1 sends $\left(r, s, t, r^{\prime}, s^{\prime}, t^{\prime}\right)$ to minus itself we may assume that we are in one of the following cases

- $t=1, r=0$ and precisely one of $\left(s, r^{\prime}, s^{\prime}\right)$ is zero.

- $t=0, t^{\prime}=1, r^{\prime}=0$ and $s, s^{\prime}$ are nonzero.

- $t=0, t^{\prime}=0$, but then also $s^{\prime}=s=0$. In this case, we have $r=1$ or $(r=0$ and $\left.r^{\prime}=1\right)$.

This leads to 52 cases.

For each of the 52 cases, we let a computer calculate the point

$$
\begin{aligned}
& {[r+2(s+t)] P_{1}+[2 r+s] \omega P_{1}+[2 r+t] P_{2}+[t] \omega P_{2}} \\
& \quad+\left[r^{\prime}+2\left(s^{\prime}+t^{\prime}\right)\right] P_{3}+\left[2 r^{\prime}+s^{\prime}\right] \omega P_{3}+\left[2 r^{\prime}+t^{\prime}\right] P_{4}+\left[t^{\prime}\right] \omega P_{4}
\end{aligned}
$$

We used then the 3-division polynomial to check whether this point is divisible by three in the Mordell-Weil group. It turned out that this is never the case.

Hence the $P_{i}$ generate the Mordell-Weil group as $\mathbf{Z}[\omega]$-module.

Proposition 2.2. The points of $E(\mathbf{C}(s, t))$ with coordinates in $\mathbf{C}[s, t]$ are precisely the points in the $\mu_{6}$-orbits of $P_{1}, P_{2}, P_{1}+P_{2}, P_{3}, P_{4}, P_{3}+P_{4}$.

Proof. The torus structures correspond to the length 4 vectors. Let $h(x, y$, $z, w)=4 w^{2}+w(2 x-4 y-4 z)+4 x^{2}-4 x(y+z)+4 y^{2}+2 y z+4 z^{2}$.

Now the height of $\left(a_{1}, \ldots, a_{8}\right)$ equals $h\left(a_{1}, a_{2}, a_{3}, a_{4}\right)+h\left(a_{5}, a_{6}, a_{7}, a_{8}\right)$. Since the shortest vectors in the lattice have length 4 , and the lattice is a direct sum, we have that any length 4 vector satisfies $a_{1}=a_{2}=a_{3}=a_{4}=0$ or $a_{5}=a_{6}=a_{7}=a_{8}=0$.

By completing squares, we find that

$$
h(x, y, z, w)=4\left(w+\frac{1}{4} x-\frac{1}{2} y-\frac{1}{2} z\right)^{2}+3\left(z-\frac{1}{2} x\right)^{2}=3\left(x-\frac{1}{2} y\right)^{2}+\frac{9}{4} y^{2}
$$

Hence to have a length 4 vector we need $9 / 4 y^{2} \leq 4$. This yields that $y$ is in $\{-1,0,1\}$. Repeating the same argument for the other coordinates we find that each of $x, y, z, w \in\{-1,0,1\}$. One calculates easily which vectors occur. It turns out that there are 18 vectors of length 4 . Up to the $\mu_{6}$-action we get that the length 4 vectors correspond with $(1,0,0,0),(0,0,1,0)$ and $(1,0,1,0)$.

Hence the inequivalent torus structures correspond to $P_{1}, P_{2}, P_{1}+P_{2}, P_{3}, P_{4}$, $P_{3}+P_{4}$.

Remark 2.3. Recall that $x_{1}, \ldots, x_{6}$ are precisely the $x$-coordinates of $P_{1}, P_{2}, P_{3}$, $P_{4}, P_{1}+P_{2}, P_{3}+P_{4}$. Hence their knowledge suffices to obtain explicit equations for all torus structures. 


\subsection{Galois action}

All the $P_{i}$ are defined over $\mathbf{Q}[\omega][u, v, w]$. We would like to find all points with coordinates in $\mathbf{Q}[u, v, w]$. For this, we need to know the $\operatorname{Gal}(\mathbf{Q}[\omega] / \mathbf{Q})$ representation on the Mordell-Weil group. First, note that the generator $\sigma$ of the Galois group is an involution, so all eigenvalues of $\sigma$ acting on the vector space $E(\mathbf{Q}[\omega](s, t)) \otimes_{\mathbf{z}} \mathbf{Q}$ are 1 and -1 . We start by determining the 1-eigenspace of $E(\mathbf{Q}[\omega](s, t)) \otimes_{\mathbf{Z}} \mathbf{Q}$.

Lemma 2.4. We have $E(\mathbf{Q}(s, t)) \cong \mathbf{Z}^{4}$.

Proof. Consider the Galois action on $E(\overline{\mathbf{Q}}(s, t)) \otimes \mathbf{Q}$. Let $\sigma$ be the generator of the Galois group. Let $v$ be an eigenvector for $\sigma$ for the eigenvalue 1 . Then, after clearing denominators, we may assume that $v$ has integral coordinates and corresponds to a point $P$ in $E(\mathbf{Q}(s, t))$. Since $P=(x(s, t), y(s, t))$ with $x(s, t), y(s, t) \in \mathbf{Q}(s, t)$ we have that $\omega P=(\omega x(s, t), y(s, t))$. Hence $\sigma(\omega(P))=\omega^{2} P$. The latter equals $-P-\omega P$.

Let $v$ now be an eigenvector for the eigenvalue -1 . Then, after clearing denominators, we may assume that $v$ has integral coordinates and corresponds to a point $P$ in $E(\mathbf{Q}(s, t))$. Write $P=(x(s, t), y(s, t))$ with $x(s, t), y(s, t) \in \mathbf{Q}(\omega)(s, t)$. Since $\sigma(P)=-P$, we have that $\sigma(x(s, t))=x(s, t)$ and $\sigma(y(s, t))=-y(s, t)$. In particular $\sigma(\omega P)=\sigma((\omega x(s, t), y(s, t)))=\left(\omega^{2} x(s, t),-y(s, t)\right)$. Hence $\sigma(\omega P)=-\omega^{2} P$. Hence we have on both eigenspaces that $\sigma \omega=\omega^{2} \sigma$. From this it follows that if $v_{1}, \ldots, v_{n}$ is a basis for the 1-eigenspace then $(1+2 \omega) v_{1}, \ldots,(1+2 \omega) v_{n}$ generate an $n$-dimensional subspace of the -1-eigenspace, and if $w_{1}, \ldots, w_{m}$ form a basis for the -1-eigenspace then $(1+2 \omega) w_{1}, \ldots,(1+2 \omega) w_{n}$ generate an $n$-dimensional subspace of the 1-eigenspace. In particular, both eigenspaces have the same dimension. Hence the $\sigma$-invariant part of $E(\overline{\mathbf{Q}}(s, t)) \otimes \mathbf{Q}$ has dimension 4 . Since $E(\overline{\mathbf{Q}}(s, t))$ is free it follows that $E(\mathbf{Q}(s, t))$ has rank 4 .

Proposition 2.5. The points $P_{1}, P_{2}, P_{3}-P_{4}, \quad P_{3}+\omega P_{3}+\omega P_{4}$ generate $E(\mathbf{Q}(s, t))$.

Proof. From the explicit expressions for $P_{1}$ and $P_{2}$ it follows that they are in $E(\mathbf{Q}(s, t))$. The fact that $P_{3}-P_{4}$ and $P_{3}+\omega P_{3}+\omega P_{4}$ are in $E(\mathbf{Q}(s, t))$ can be checked by directly computing these points using the group law.

One can shorten the calculations a bit by the following observations: A straightforward calculation shows that $Q:=P_{3}+P_{4}$ is of the form $(x(s, t), \sqrt{-3} y(s, t))$ with $x(s, t), y(s, t) \in \mathbf{Q}(s, t)$. Hence $\sigma(Q)=-Q$ and by the proof of the previous lemma $R:=(1+2 \omega) Q$ is invariant under $\sigma$.

Similarly, $S:=P_{3}-P_{4}$ is of the form $(x(s, t), y(s, t))$ with $x(s, t), y(s, t) \in \mathbf{Q}(s, t)$. Hence $R+S=2\left(P_{3}+\omega P_{3}+\omega P_{4}\right)$ is rational. Clearly, $P_{3}+\omega P_{3}+\omega P_{4} \in E(\mathbf{Q}(\omega)(s, t))$. 
Let $\sigma \in \operatorname{Gal}(\mathbf{Q}(\omega) / \mathbf{Q})$. Then $2\left(\sigma\left(P_{3}+\omega P_{3}+\omega P_{4}\right)\right)=2\left(P_{3}+\omega P_{3}+\omega P_{4}\right)$. Since $E(\overline{\mathbf{Q}}(s, t))$ is torsion-free it follows that $\sigma\left(P_{3}+\omega P_{3}+\omega P_{4}\right)=P_{3}+\omega P_{3}+\omega P_{4}$. Therefore $P_{3}+\omega P_{3}+\omega P_{4} \in E(\mathbf{Q}(s, t))$.

The matrix

$$
\left(\begin{array}{cccccccc}
1 & 0 & 0 & 0 & 0 & 0 & 0 & 0 \\
0 & 0 & 1 & 0 & 0 & 0 & 0 & 0 \\
0 & 0 & 0 & 0 & 0 & -1 & 0 & 1 \\
0 & 0 & 0 & 0 & 1 & 0 & 1 & 1
\end{array}\right)
$$

has clearly rank 4 , so these points are independent.

The points $\omega P_{1}, \omega P_{2}, P_{4}, \omega P_{4}$ and the above four points generate $E(\mathbf{C}(s, t))$. In particular, each subset of this set generates a saturated subgroup of $E(\overline{\mathbf{Q}}(s, t))$. In particular, $P_{1}, P_{2}, P_{3}-P_{4}$ and $P_{3}+\omega P_{3}+\omega P_{4}$ generate $E(\mathbf{Q}(s, t))$.

Remark 2.6. The points $P_{1}$ and $P_{2}$ have height 4 . The point $P_{3}-P_{4}$ has height 12 , the point $P_{3}+\omega P_{3}+\omega P_{4}$ has height 6 .

\section{How to find the $P_{i}$}

In this final section, we describe which methods we used to find $P_{1}, P_{2}, P_{3}, P_{4}$.

\section{1. $P_{1}, P_{2}$}

Note that $f:=g\left(u^{3}, v^{3}, w^{3}\right)$ for some quartic polynomial $g$. A quartic curve cannot have a $(2,3)$-torus structure. However, sextics may have such a structure. Now reducible sextics that are reduced do not have $(2,3)$-torus structure $[5$, Theorem $34]$, but non-reduced reducible sextics may have them.

It turns out that $u^{2} g$ has various torus structures. A torus structure of $u^{2} g$ can be pulled back to a torus structure of $f$ if and only if the $x$ - and the $y$-coordinate are both divisible by $u$. Now setting $x=u \ell$ and $y=u c$ with $\ell$ a generic linear form and $c$ a generic quadric and solving $y^{2}=x^{3}-1296 u^{2} g$ yields 15 equations in 9 unknowns. This system can be easily solved by a computer algebra package.

One of the solutions is $x=-36 u(u+w)$, then $x^{3}-1296 u^{2} g$ is a square $y^{2}$ with

$$
y=36 u\left(3 u^{2}+u(-6 v+12 w)+v^{2}+w^{2}+v w\right)
$$

If we now substitute $u=u^{3}, v=v^{3}, w=w^{3}$ in this torus structure then we find a $(2,3)$-torus structure of $-1296 u^{6} f$. Since both $x$ and $y$ are divisible by $u^{3}$ we can divide $x$ by $u^{2}$ and $y$ by $u^{3}$ and we find a torus structure of $-1296 f$.

Swapping $v$ and $w$ gives another torus structure.

This procedure yields the points $P_{1}, P_{2}$. 


\section{2. $P_{3}, P_{4}$}

We can find a further generator as follows. The equation is symmetric in $v$ and $w$. Hence there is a polynomial $h$ in $s$ and $t$ of weighted degree 12 (with $\operatorname{deg}(s)=1, \operatorname{deg}(t)=2)$ such that $f(1, v, w)=h(v+w, v w)$.

We can then consider the degree 12 curve $r^{12} h(s / r, t / r)=0$. Using a computer algebra package, one easily finds two torus structures. The point $P_{1}+P_{2}$ is clearly symmetric in $v$ and $w$ and is the pullback of one the torus structures. The pull back of the other torus structure has $x$-coordinate

$$
-12\left(v^{4}+w^{4}-v^{3} w-v w^{3}+3 v^{2} w^{2}-3 u^{3}(v+w) .\right.
$$

Call this point $P_{7}$.

Apply now $v \mapsto \omega v$ to $P_{7}$. The obtained torus structure is one of $\pm \omega^{2} P_{3}$. Similarly, apply now $w \rightarrow \omega w$ to $P_{5}$. Then we obtain one of $\pm \omega^{2} P_{4}$. In order to simplify several formulas, we decided to work with $P_{3}$ and $P_{4}$ rather than with the points obtained here.

\section{References}

1. Cogolludo-Agustín, J.-I. and Libgober, A., Mordell-Weil groups of elliptic threefolds and the Alexander module of plane curves, J. Reine Angew. Math. 697 (2014), 15-55.

2. Degtyarev, A., Topology of Algebraic Curves, De Gruyter Studies in Mathematics 44, Walter de Gruyter \& Co., Berlin, 2012.

3. Hirano, A., Construction of plane curves with cusps, Saitama Math. J. 10 (1992), $21-24$.

4. Kloosterman, R., Mordell-Weil lattices and toric decompositions of plane curves, Math. Ann. 367 (2017), 755-783.

5. Ока, M., A survey on Alexander polynomials of plane curves, in Singularités FrancoJaponaises, Sémin. Congr. 10, pp. 209-232, Soc. Math. France, Paris, 2005.

6. Pнo, D. T., Alexander polynomials of certain dual of smooth quartics, Proc. Japan Acad. Ser. A Math. Sci. 89 (2013), 119-122.

Remke Kloosterman

Dipartimento di Matematica

Università degli Studi di Padova

Via Trieste 63

35121 Padova

Italy

klooster@math.unipd.it

Received January 26, 2017

in revised form January 9, 2018 